\title{
Molecular cloning and characterization of the pseudorabies virus US1 gene
}

\author{
M.L. Li ${ }^{1}$, J.H. Chen ${ }^{2}$, Z.Y. Zhao ${ }^{1}$, K.J. Zhang ${ }^{1}$, Z. Li ${ }^{1}$, J. Li ${ }^{1}$, J.Y. Mai ${ }^{1}$, \\ X.M. Zhu ${ }^{1}$ and M.S. Cai ${ }^{1,2}$ \\ ${ }^{1}$ Department of Pathogenic Biology and Immunology, \\ Guangzhou Medical University, Guangzhou, China \\ ${ }^{2}$ Department of Veterinary Medicine, \\ Foshan Science and Technology University, Foshan, China \\ Corresponding author: M.S. Cai \\ E-mail: mingshengcai@hotmail.com
}

Genet. Mol. Res. 12 (1): 85-98 (2013)

Received July 3, 2012

Accepted September 3, 2012

Published January 22, 2013

DOI http://dx.doi.org/10.4238/2013.January.22.7

\begin{abstract}
Using polymerase chain reaction, a 1050-bp sequence of the US1 gene was amplified from the pseudorabies virus (PRV) Becker strain genome; identification of the US1 gene was confirmed by further cloning and sequencing. Bioinformatics analysis indicated that the PRV US1 gene encodes a putative polypeptide with 349 amino acids. The encoded protein, designated PICP22, had a conserved Herpes_IE68 domain, which was found to be closely related with the herpes virus immediate early regulatory protein family and is highly conserved among the counterparts encoded by Herpes_IE68 genes. Multiple nucleic acid sequence and amino acid sequence alignments suggested that the product of PRV US1 has a relatively higher homology with ICP22like proteins of genus Varicellovirus than with those of other genera of Alphaherpesvirinae. In addition, phylogenetic analysis showed that PRV US1 has a close evolutionary relationship with members of the genus Varicellovirus, especially Equid herpes virus 1 (EHV-1), EHV-4 and EHV-9. Antigen prediction indicated that several potential B-cell epitopes are located in PICP22. Also, subcellular localization analysis demonstrated that PICP22 is predominantly located in the cytoplasm,
\end{abstract}


suggesting that it might function as a cytoplasmic-targeted protein.

Key words: Pseudorabies virus; US1; ICP22; Cloning; Bioinformatics; Molecular characterization

\section{INTRODUCTION}

Aujeszky's disease, which is caused by pseudorabies virus (PRV), is a frequently fatal disease with a global distribution that primarily affects swine and other domestic and wild animals incidentally. PRV belongs to the genus Varicellovirus, subfamily Alphaherpesvirinae, which is a swine alphaherpesvirus closely related to the human pathogen herpes simplex virus 1 and 2 (HSV-1 and HSV-2) and varicella-zoster virus (VZV) (Kramer et al., 2011). The neurotropic nature of PRV makes it a useful tracer of neuronal pathways and it is a useful model for the study of herpesvirus pathogenesis. PRV has caused great economic losses in the pig industry worldwide, while efforts to eradicate PRV have shown great progress, it remains an endemic problem in many countries (Kramer et al., 2011).

To understand the fundamental mechanisms underlying PRV spread and pathogenesis, it is particularly important to have a comprehensive understanding of the function of each gene in the course of viral replication. During the lytic cycle of PRV infection, viral genes are expressed in a cascade of 3 temporally distinct and functionally interdependent phases termed immediate-early (IE), early (E), and late (L). Although several PRV genes, including the unique IE gene IE180 (Tomioka et al., 2008), 3 E genes EP0 (Brukman and Enquist, 2006), UL54 (Li et al., 2011a,b), and UL23 (Ferrari et al., 2000), and many L genes such as capsid genes UL25 (Coller et al., 2007) and UL35 (Krautwald et al., 2008), tegument genes UL36, UL37 (Luxton et al., 2006), and UL41 (Lin et al., 2010), and envelope genes UL27 (Nixdorf et al., 2001a), UL44 (Kramer et al., 2011), US8, and UL10 (Nixdorf et al., 2001b), have been extensively studied, the function of US1 and its protein product ICP22 (PICP22) is less well understood.

In this study, the US1 gene was amplified from the PRV Becker genome using polymerase chain reaction (PCR) followed by cloning and sequencing. A comprehensive bioinformatics analysis was conducted to study the molecular characteristics of US1 and to provide molecular biological insight for future study on the function and mechanism of PICP22 during PRV infection. The analysis included a large number of bioinformatics tools such as open reading frame (ORF) Finder, Conserved Domains, DNAstar 7.0, Bioedit 7.0, SignalP-4.0, NetPhos 2.0, PSIpred, CPHmodels 3.2, and PSORT II.

\section{MATERIAL AND METHODS}

\section{Cloning and sequencing of PRV US1}

PCR amplification primers for US1 (accession No. JF797219) were designed using Oligo 6.0 and Primer 5.0; reactions were performed with TaKaRa reagents. The upstream primer 5'-TTGAATTCATGGACCGGGACCGGGCCTG-3' anneals to the first 20 nucleotides of US1 and introduces an EcoRI restriction site (underlined) for cloning. The downstream primer 5'-AACTCGAGTCACGGGGCGGCGGCGGG-3' is complementary to the final 18 nucleotides of USI and introduced an XhoI restriction site (underlined). 
The US1 gene was PCR-amplified from the genomic DNA of PRV Becker, previously purified from vBecker2-infected PK-15 cells (Smith and Enquist, 2000; Li et al., 2011a,b). PCR profiles involved initial predenaturation for $5 \mathrm{~min}$ at $95^{\circ} \mathrm{C}$ followed by 30 cycles of denaturation at $94^{\circ} \mathrm{C}$ for $50 \mathrm{~s}$, annealing at $65^{\circ} \mathrm{C}$ for $40 \mathrm{~s}$, and extension at $72^{\circ} \mathrm{C}$ for 1 min $15 \mathrm{~s}$. The final extension step was performed at $72^{\circ} \mathrm{C}$ for $10 \mathrm{~min}$. Purified PCR product was digested with $E c o \mathrm{RI}$ and $X h o \mathrm{I}$ and ligated into pre-digested prokaryotic expression vector pET28a $(+)$ (Novagen) to generate pET28a(+)-US1. Presence of the appropriate insert was verified by $\mathrm{PCR}$, restriction analysis, and sequencing.

\section{Bioinformatics analysis of the nucleotide sequence of PRV US1}

To determine nucleotide sequence similarity and identify the ORF, we used NCBI BLASTN (http://www.ncbi.nlm.nih.gov/BLAST/) and ORF Finder (http://www.ncbi.nlm.nih. gov/gorf/gorf.html). Subsequently, Clustal V in the MegAlign program of DNAStar (version 7.0, DNAStar, Inc.) was used to analyze nucleotide sequence homology of 19 ICP22-like proteins of alphaherpesvirus (listed in Table 1).

\section{Bioinformatics analysis of the deduced amino acid sequence of PRV US1}

For amino acid (aa) sequence comparison, homology search, and conserved domain analysis, the aa sequence of PICP22 was analyzed using BLASTP (http://www.ncbi.nlm.nih. gov/BLAST/) and the Conserved Domains search tool (http://www.ncbi.nlm.nih.gov/Structure/ cdd/wrpsb.cgi). To compare PICP22 with ICP22-like proteins of other alphaherpesviruses (listed in Table 1), aa sequence homology and phylogenetic relationships were performed in DNAstar 7.0. To predict the signal peptide sequence, transmembrane domain, glycosylation site, phosphorylation site, and hydrophobic and hydrophilic regions, B-cell epitope, secondary structure, 3-dimensional (3-D) structure, and subcellular localization of PICP22, SignalP-4.0 Server (http://www.cbs.dtu.dk/services/SignalP/), TMHMM (http://www.cbs.dtu.dk/services/ TMHMM/), NetNGlyc 1.0 (http://www.cbs.dtu.dk/services/NetNGlyc/), NetPhos 2.0 (http:// www.cbs.dtu.dk/services/NetPhos/), Bioedit 7.0, DNAstar 7.0, PSIpred (http://bioinf.cs.ucl. ac.uk/psipred/), CPHmodels 3.2 (http://www.cbs.dtu.dk/services/CPHmodels/), and PSORT (http://psort.nibb.ac.jp/) were used.

\section{RESULTS}

\section{PCR amplification and cloning of PRV US1}

The US1 gene was PCR-amplified from the purified genome of PRV Becker. As shown in Figure 1, no specific band was amplified from the mock-infected control (Figure 1, lane 1), whereas a target fragment of $1050 \mathrm{bp}$, consistent with the expected size, was amplified from DNA purified from PRV-infected PK-15 cells (Figure 1, lane 2). The DNA fragment was cloned into prokaryotic expression vector pET28a $(+)$ to yield pET28a $(+)$-US1 (Figure 1, lane 3 ), which was confirmed by restriction analysis (Figure 1, lanes 4 and 5), PCR amplification (Figure 1, lane 6), and DNA sequencing (Figure 2A). 

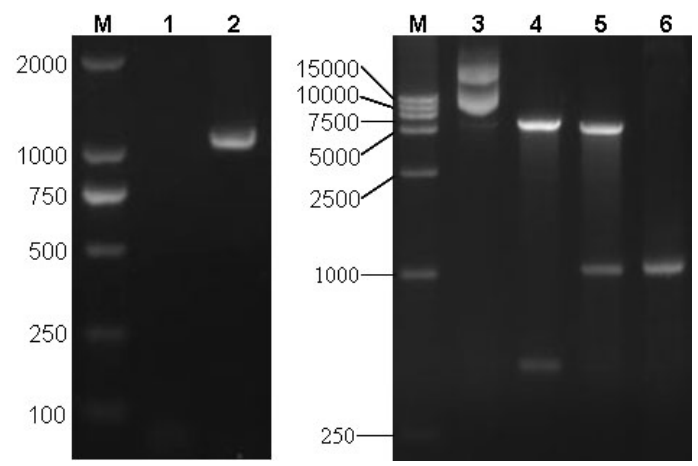

Figure 1. PCR amplification and restriction enzyme analysis of the recombinant plasmid pET28a(+)-US1. Lanes 1 and 2 = PCR amplification product of the US1 gene using DNA purified from mock- and PRV-infected PK15 cells as the template, respectively; lane $3=$ the recombinant plasmid pET28a(+)-US1; lane $4=$ restriction enzyme digestion product of pET28a(+)-US1 with EcoRI and SalI; lane $5=$ restriction enzyme digestion product of pET28a(+)-US1 with EcoRI and XhoI; lane $6=$ PCR amplification product of the US1 gene from pET28a(+)-US1. Samples were electrophoresed through a $1 \%$ agarose gel and stained with ethidium bromide. The electrophoresis migration of molecular mass marker (lane $M, \mathrm{TaKaRa}$ ) was also shown.
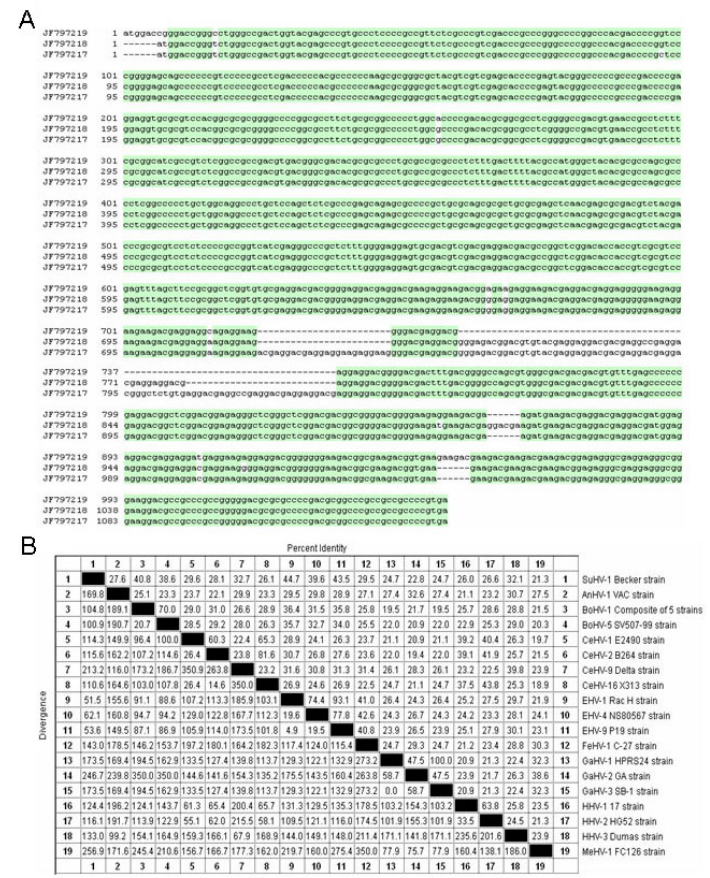

Figure 2. Multiple nucleic acid sequence alignment of the US1 gene of the PRV Becker strain with other PRV strains and its homologous genes of 18 different selected species. A. Multiple nucleic acid sequence alignment of the US1 gene of the PRV Becker strain (JF797219) with Kaplan (JF797218) and Bartha strains (JF797217) using Clone Manger (version 8). B. Multiple nucleic acid sequence alignment of the US1 gene of the PRV Becker strain with its homologous genes of 18 selected species (Table 1) by using the MEGALIGN program in LASERGENE (DNAStar 7.0) with the Clustal V method, and sequence distance was calculated using weight matrix Identity. Gaps had been introduced by the alignment program to maximize the homology. 


\section{Bioinformatics analysis of the PRV US1 nucleotide sequence}

ORF Finder analysis revealed an integrated PRV US1 ORF consisting of $1050 \mathrm{bp}$. Nucleotide sequence similarity search by NCBI BLASTN yielded 2 nucleotide sequences (accession Nos. JF797218 and JF797217) with strong similarity to PRV Becker US1 (similarity up to 92 and $89 \%$, respectively) (Figure 2A); these accessions correspond to the US1 gene of the PRV Kaplan and Bartha strains. Multiple alignment of PRV US1 with 18 homologous reference alphaherpesviruses (Table 1) revealed remarkably high homology of 32.1 to $44.7 \%$ with the members of genus Varicellovirus, subfamily Alphaherpesvirinae, i.e., Human herpesvirus 3 (HHV-3, VZV), Cercopithecine herpesvirus 9 (CeHV-9), Bovine herpesvirus 5 (BoHV-5), Equine herpesvirus 4 (EHV-4), BoHV-1, EHV-9, and EHV-1. However, low homology was detected between PRV and other viruses of the subfamily Alphaherpesvirinae (Figure 2B).

\begin{tabular}{|c|c|c|c|c|}
\hline Genus & Virus name (Abbreviation) & Strain & Natural host & $\begin{array}{l}\text { GenBank } \\
\text { accession No. }\end{array}$ \\
\hline \multirow[t]{18}{*}{ Varicellovirus } & \multirow{3}{*}{$\begin{array}{l}\text { Suid herpesvirus } 1 \text { (SuHV-1) } \\
\text { Pseudorabies virus (PRV) }\end{array}$} & Becker & \multirow[t]{3}{*}{ Sus scrofa (Pig) } & AEM64105 \\
\hline & & Kaplan & & AEM64036 \\
\hline & & Bartha & & AEM63971 \\
\hline & Equid herpesvirus 1 (EHV-1) & \multirow[t]{2}{*}{ Rac H } & \multirow[t]{2}{*}{ Equus caballus (Horse) } & \multirow{2}{*}{ CAA91927 } \\
\hline & Equine abortion virus (EAV) & & & \\
\hline & Equine herpesvirus 4 (EHV-4) & \multirow[t]{2}{*}{ NS80567 } & \multirow{2}{*}{ Equus caballus (Horse) } & \multirow{2}{*}{ NP_045295 } \\
\hline & Equine rhinopneumonitis virus (ERV) & & & \\
\hline & Equid herpesvirus 9 (EHV-9) & \multirow[t]{2}{*}{ P19 } & \multirow[t]{2}{*}{ Equus caballus (Horse) } & \multirow[t]{2}{*}{ YP_002333546 } \\
\hline & Gazelle herpesvirus 1 (GHV-1) & & & \\
\hline & Cercopithecine herpesvirus 9 (CeHV-9) & \multirow[t]{2}{*}{ Delta } & \multirow[t]{2}{*}{ Erythrocebus patas (Monkey) } & \multirow[t]{2}{*}{ NP_077477 } \\
\hline & Simian varicella virus (SVV) & & & \\
\hline & Felid herpesvirus 1 (FeHV-1) & $\mathrm{C}-27$ & Felidae (Cat) & YP_003331584 \\
\hline & Bovine herpesvirus 1 (BoHV-1) & \multirow{2}{*}{$\begin{array}{l}\text { Composite } \\
\text { of } 5 \text { strains }\end{array}$} & \multirow[t]{2}{*}{ Bos taurus (Cattle) } & \multirow[t]{2}{*}{ NP_045365 } \\
\hline & Infectious bovine rhinotracheitis virus (IBRV) & & & \\
\hline & Bovine herpesvirus 5 (BoHV-5) & \multirow[t]{2}{*}{ SV507/99 } & \multirow[t]{2}{*}{ Bos taurus (Cattle) } & \multirow{2}{*}{ YP_003662528 } \\
\hline & Bovine encephalitis herpesvirus (BEHV) & & & \\
\hline & Human herpesvirus 3 (HHV-3) & \multirow[t]{2}{*}{ Dumas } & \multirow[t]{2}{*}{ Homo sapiens (Human) } & \multirow[t]{2}{*}{ NP_040185 } \\
\hline & Varicella-zoster virus (VZV) & & & \\
\hline \multirow[t]{10}{*}{ Simplexvirus } & Human herpesvirus 1 (HHV-1) & \multirow[t]{2}{*}{17} & \multirow[t]{2}{*}{ Homo sapiens (Human) } & \multirow[t]{2}{*}{ CAA32287 } \\
\hline & Herpes simplex virus 1 (HSV-1) & & & \\
\hline & Human herpesvirus 2 (HHV-2) & HG52 & Homo sapiens (Human) & CAB06708 \\
\hline & Herpes simplex virus 2 (HSV-2) & & & \\
\hline & Cercopithecine herpesvirus 1 (CeHV-1) & E2490 & Macaca mulatta (Monkey) & BAB83748 \\
\hline & Macacine herpesvirus 1 (McHV-1) & & & \\
\hline & Monkey B virus & & & \\
\hline & $\begin{array}{l}\text { Cercopithecine herpesvirus } 2 \text { (CeHV-2) } \\
\text { Simian agent } 8 \text { (SA8) }\end{array}$ & B264 & Cercopithecus aethiops (Monkey) & YP_164503 \\
\hline & Cercopithecine herpesvirus 16 (CeHV-16) & $\mathrm{X} 313$ & Papio cynocephalus (Baboons) & ABA29314 \\
\hline & Papiine herpesvirus 2 (PaHV-1) & & & \\
\hline Iltovirus & Gallid herpesvirus 1 (GaHV-1) & HPRS24 & White Leghorn (Chicken) & BAA32006 \\
\hline & Infectious laryngotrach eitis virus (ILTV) & & & \\
\hline Mardivirus & Gallid herpesvirus 2 (GaHV-2) & GA & Gallus domesticus (Chicken) & AAT65010 \\
\hline & Marek's disease virus type 1 (MDV-1) & & & \\
\hline & Gallid herpesvirus 3 GaHV-3) & SB-1 & Gallus gallus (Chicken) & AEI00291 \\
\hline & Marek's disease virus type 2 (MDV-2) & & & \\
\hline & Meleagrid herpesvirus 1 (MeHV-1) & FC126 & Meleagris gallopavo (Turkey) & AF282130_68 \\
\hline & Anatid herpesvirus 1 (AnHV-1) & $\mathrm{VAC}$ & Anatid species (Duck) & YP 003084427 \\
\hline & Duck enteritis virus (DEV) & & & \\
\hline
\end{tabular}




\section{Bioinformatics analysis of the PICP22 polypeptide sequence}

An aa sequence similarity search in NCBI BLASTP yielded 2 aa sequences (accession Nos. AEM64036 and AEM63971) that strongly matched the target sequence of PRV Becker ICP22 (similarity up to 92 and 88\%, respectively); these correspond to the ICP22 protein of the PRV Kaplan and Bartha strains, respectively (Figure 3A). Multiple sequence alignment of PICP22 with its homologs in 18 reference alphaherpesviruses (Table 1) showed relatively high homology of 30.3 to $38.8 \%$ between PICP22 and its BoHV-1, EHV-4, EHV-9, and EHV-1 counterparts. However, PICP22 shared no substantial homology with ICP22-like proteins from HHV-2 (HSV-2), HHV-1 (HSV-1), CeHV-16, CeHV-1, or CeHV-2 (16.9 to 17.8\%; Figure 3B). Conserved domain analysis indicated that PICP22 contains a conserved domain of Herpes IE68 (Figure 3C), which is an immediate early protein. Further searches revealed 13 conserved domains (data not shown), all similar to Herpes_IE68. Thus, PICP22 is closely related to the immediate early protein family and is similar to its counterparts encoded by Herpes_IE68 genes. Therefore, PICP22 might belong to the Herpes_IE68 family.

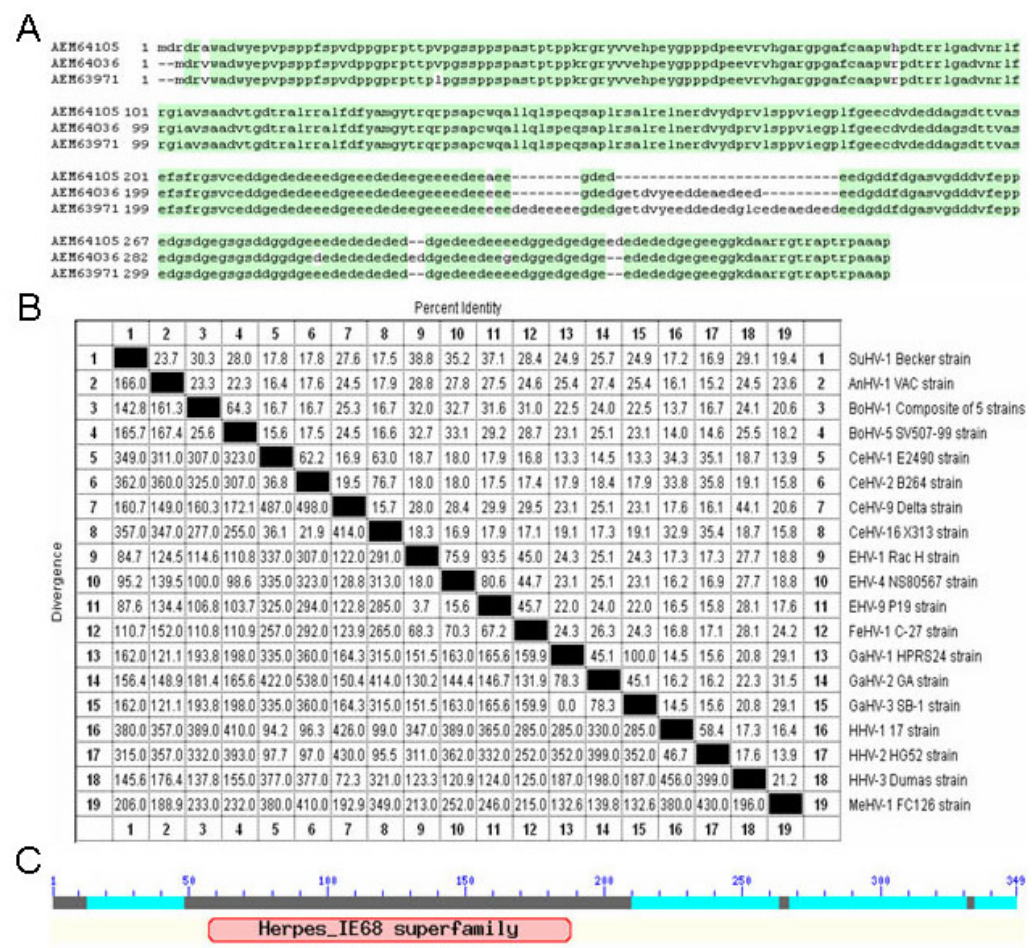

Figure 3. Conserved domain analysis and multiple aa sequence alignment of the PICP22 of PRV Becker strain with other PRV strains and its homologous proteins of 18 different selected species. A. Multiple aa sequence alignment of PICP22 of the PRV Becker strain (AEM64105) with Kaplan (AEM64036) and Bartha strains (AEM63971) using Clone Manger (version 8). B. Multiple aa sequence alignment of PICP22 of the PRV Becker strain with its homologous genes of 18 selected species (Table 1) by using the MEGALIGN program in LASERGENE (DNAStar 7.0) with the Clustal V method, and sequence distance was calculated using weight matrix PAM250. Gaps had been introduced by the alignment program to maximize the homology. C. Conserved domain analysis of PRV ICP22 by using NCBI Conserved Domains search tool. 
Phylogenetic analysis of PRV and other herpesviruses was performed based on the sequences of PICP22 and the ICP22-like proteins of 18 reference alphaherpesviruses (Table 1). The proteins could be preliminarily separated into different genera, i.e., Simplexvirus, Varicellovirus, Mardivirus, and Iltovirus. This was consistent with the existing classification of subfamily Alphaherpesvirinae (Figure 4). Furthermore, PICP22 clustered with EHV-4, EHV-9, and EHV-1 first, and then clustered with other varicelloviruses, including CeHV-9, HHV-3, BoHV-1, and BoHV-5 (Figure 4). Therefore, PRV might have a closer evolutionary relationship with the members of genus Varicellovirus than other genera of Alphaherpesvirinae.

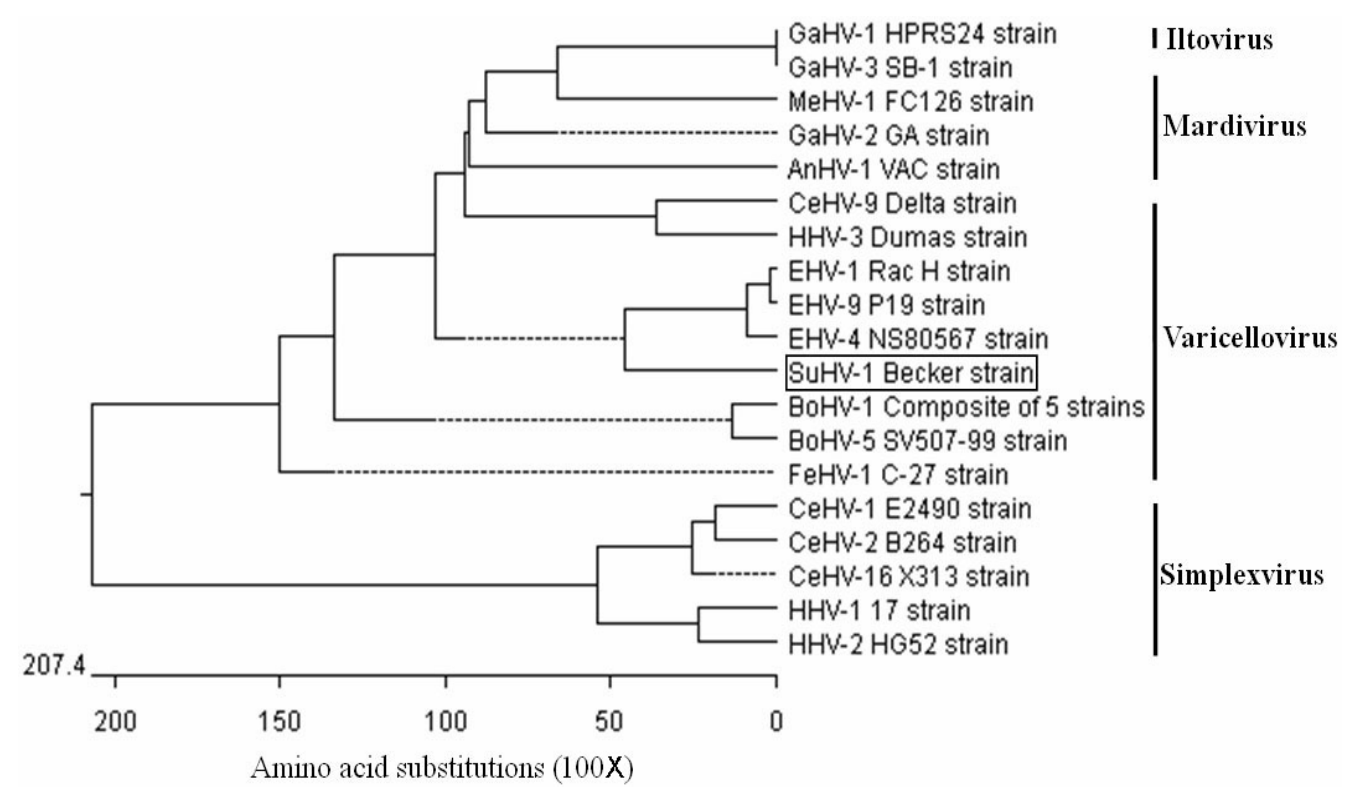

Figure 4. Evolutionary relationships of the putative PRV ICP22 protein with its 18 reference alphaherpesviruses from different species (Table 1). Phylogenetic tree of these proteins was generated by using the MEGALIGN program in LASERGENE (DNAStar 7.0) with the Clustal V method, and sequence distance was calculated using weight matrix PAM250. Gaps had been introduced by the alignment program to maximize the homology.

Signal polypeptide prediction indicated no cleavage site (Figure 5A) and no transmembrane domain in PICP22 (Figure 5B). N-linked glycosylation site (Asn-X-Ser/Thr) prediction demonstrated no N-glycosylation site (Figure 5C), which confirmed the absence of a signal peptide in PICP22. Interestingly, there were 22 potential phosphorylation sites in PICP22 (Figure 5D), including 15 serine, 5 threonine, and 2 tyrosine residues. Hydrophobicity analysis revealed 6 hydrophobic regions located at aa 73-80, 97-112, 120-125, 138-148, 168-182, and 198-205 (Figure 6A). The hydrophilic region was larger than the hydrophobic region (Figure 6B), suggesting that PICP22 might be a hydrophilic protein. 

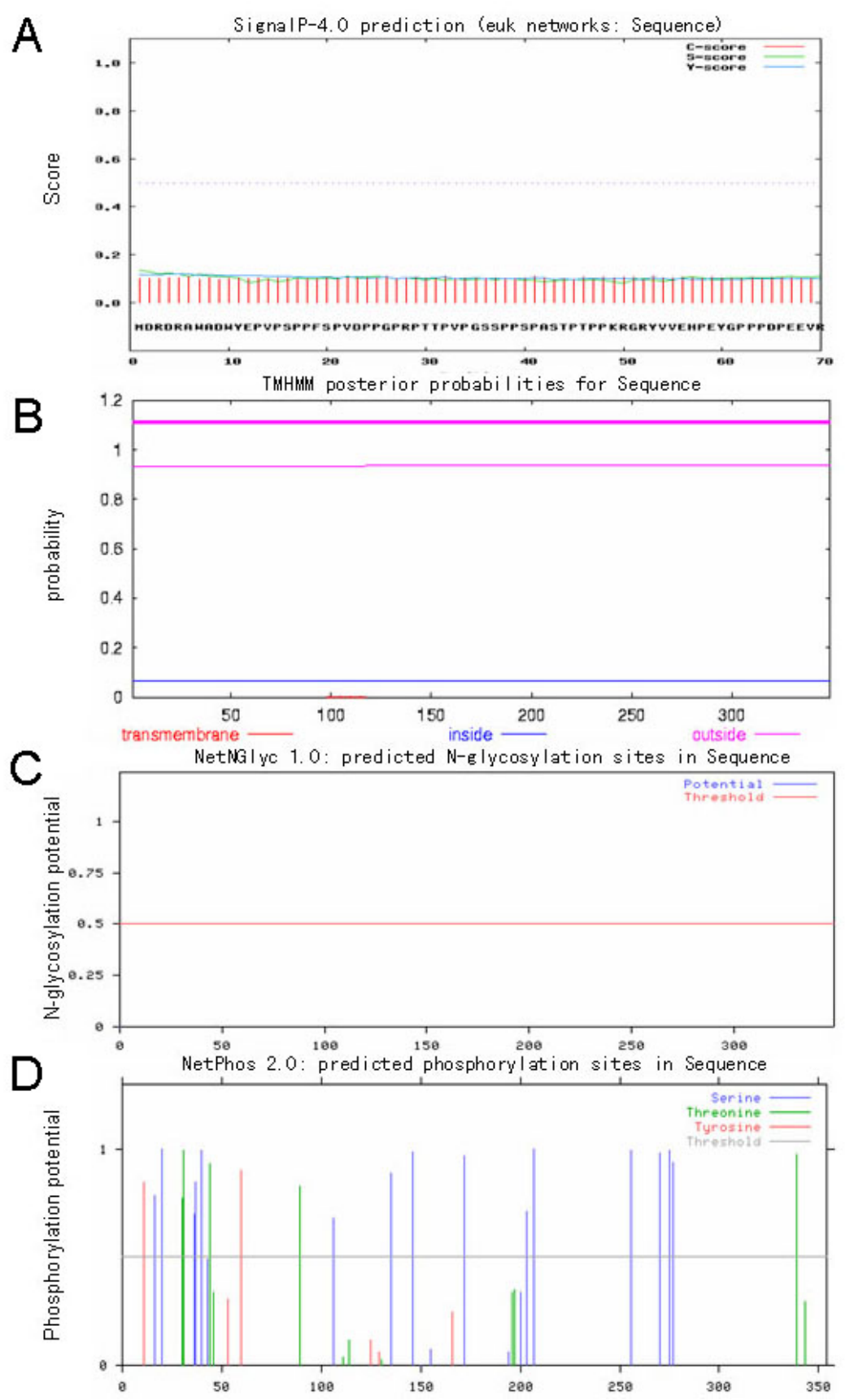

Figure 5. The prediction of signal peptide sequence, transmembrane domain, glycosylation site, and phosphorylation site of PICP22. Signal peptide sequence, transmembrane domain, glycosylation site and phosphorylation site of PICP22 were analyzed by SignalP-4.0 Server (A), the TMHMM program (B), the NetNGlyc 1.0 program (C), and the NetPhos 2.0 program $(\mathbf{D})$, respectively. 


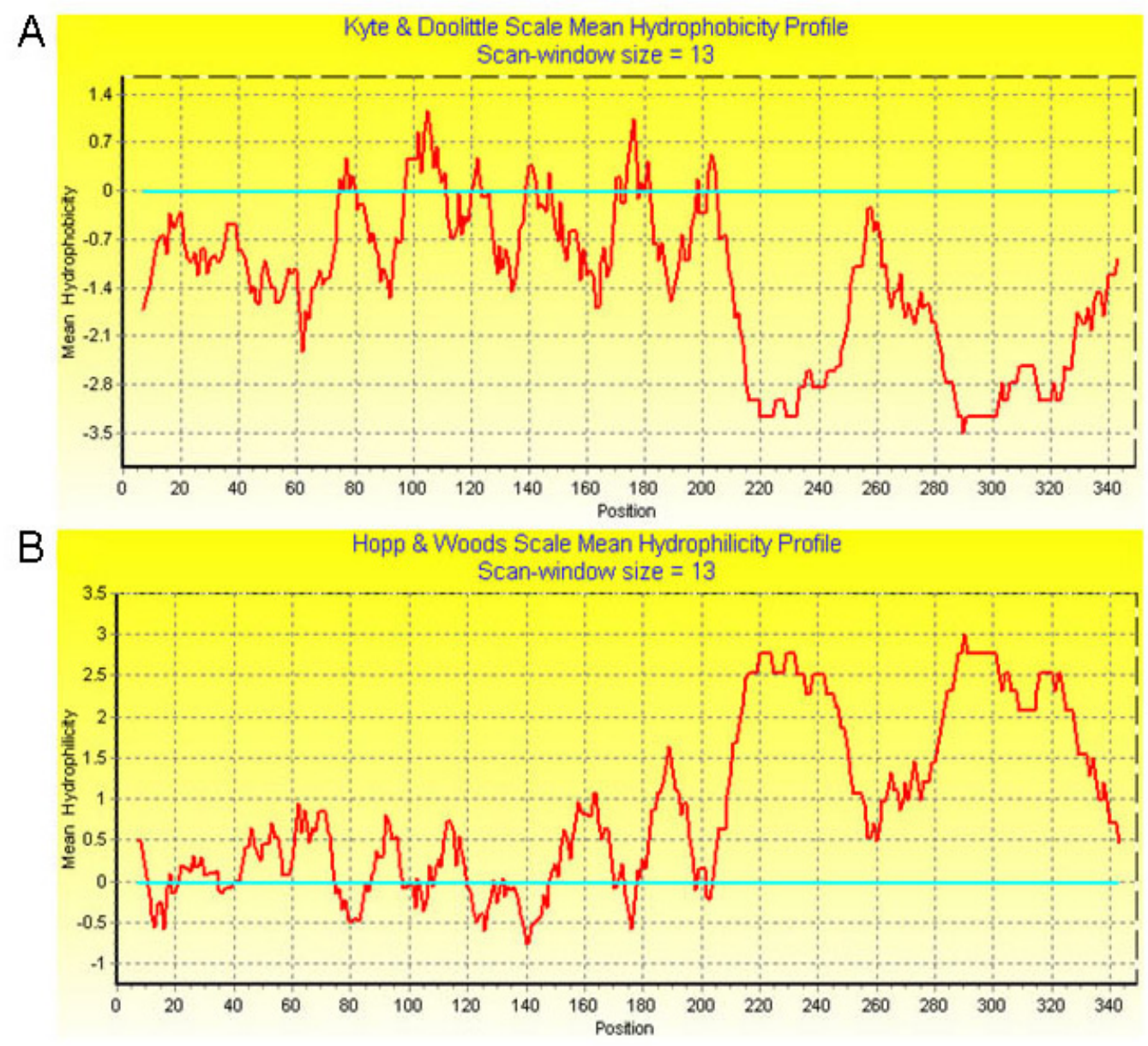

Figure 6. Hydrophobicity and hydrophilicity analyses of PICP22 by using BioEdit 7.0. The hydrophobicity (A) or hydrophilicity profile (B) was determined with the values of Kyte and Doolittle (1982) or Hopp and Woods (1981) by using a 13-amino acid window, respectively. The upwardpointing peaks represent the most hydrophobic regions (A) and the most hydrophilic regions (B), respectively.

Several potential B-cell epitopes were identified in PICP22, located in or adjacent to aa 1-8, 12-81, 84-101, 109-122, 129-138, 145-198, and 202-347 (Figure 7A). Secondary structure analysis (Figure 7B) suggests that PICP22 is primarily consisted of random coils (up to $87.39 \%$ ) and $\alpha$-helices (12.03\%); no 3-D structure model for PICP22 was found. A coiledcoil motif was predicted at 213-240 aa. Subcellular localization analysis demonstrated that PICP22 predominantly localizes to the cytoplasm $(47.8 \%)$ and nucleus $(26.1 \%)$. However, only a small fraction localized to the vacuoles $(8.7 \%)$, mitochondria $(8.7 \%)$, cytoskeleton $(4.3 \%)$, and peroxisomes $(4.3 \%)$. PICP22 is therefore predominantly located in the cytoplasm and functions as a cytoplasmic-targeted protein. 


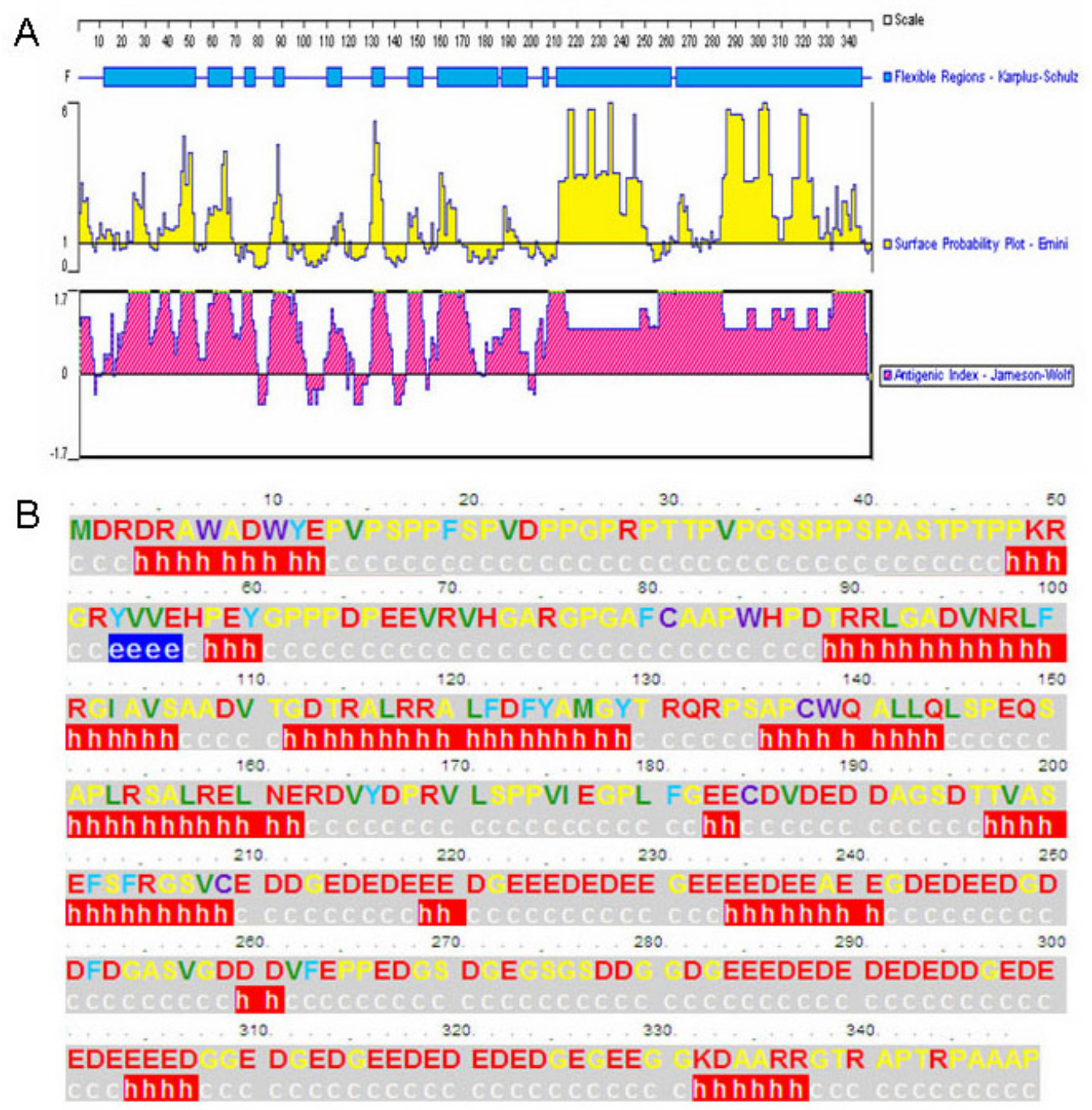

Figure 7. Antigenic analysis and secondary structure prediction of PICP22. A. Antigenic analysis of PICP22 was carried out by the PROTEAN software of DNAStar based on it flexibility, surface probability and antigenic index by the determination of its primary structure. B. Secondary structure was predicted by using the PSIpred program and the letters $\mathrm{h}, \mathrm{e}$, and $\mathrm{c}$ indicate alpha helix, extended (beta strand) and coil, respectively.

\section{DISCUSSION}

PCR amplification, cloning, and sequencing verified the presence of the US1 gene in PRV Becker. Bioinformatics analysis indicated that its encoded protein PICP22 is a member of the Herpes_IE68 family. HSV-1 ICP22 and VZV ORF63, homologs of PICP22, have been well documented; US1 likely acts as a real IE gene encoding for an IE protein in these cases. 
However, PRV US1 is not expressed as an IE gene (Zhang and Leader, 1990). Important for HSV-1 replication, ICP22 is a multifunctional protein with different roles during infection, such as inducing the formation of discrete nuclear foci containing cellular chaperone proteins known as VICE domains (Bastian et al., 2010), ensuring proper virion morphology (Orlando et al., 2006), and modulating viral and cellular gene expression (Advani et al., 2003; Bowman et al., 2009; Kalamvoki and Roizman, 2011), which are also the main functions of VZV ORF63 (Kost et al., 1995; Jones and Arvin, 2005). VZV ORF63 is also critical for efficient establishment of latency (Ambagala and Cohen, 2007). Therefore, the important roles played by HSV-1 ICP22 and VZV ORF63 in the process of infection suggest that PICP22 may play a similar role in the course of infection. However, it is not yet known which real biological functions PICP22 has in the herpesvirus life cycle and the examination of these aspects must await further study of its functions in viral replication and interactions between herpesvirus and host.

Subcellular protein localization is closely related to its functional role. Specific aa sequences determine whether a protein will span the cell membrane and enter the cell, fuse to the intramembrane, or be exported from the cell. It appears to be a universal phenomenon that almost every protein is marked with a localization signal (Feng, 2002). Subcellular localization research of HSV-1 ICP22 suggests that in an HSV-1-infected cell, ICP22 predominantly locates in the nucleus, particularly in the small, dense nuclear bodies early in infection and in the diffuse replicative complexes after onset of DNA synthesis (Jahedi et al., 1999; Xing et al., 2011). PICP22 was predicted to be localized in the cytoplasm where it might function as a cytoplasmic-targeted protein, although it does contain a putative nuclear localization signal (NLS, 47PPKRGRY53). Although VZV ORF63 contains a functional NLS, it accumulates exclusively in the cytoplasm of latently infected sensory neurons; it shifts to nuclear and cytoplasmic localization following reactivation from latency (Walters et al., 2008). Therefore, PICP22 might have the same subcellular localization pattern as VZV ORF63, since PICP22 was more similar to VZV ORF63 than to HSV-1 ICP22. However, this inference has not been verified.

Protein phosphorylation is one of the most normal and essential types of protein modification; certain aspects of cell process modulation are regulated by protein phosphorylation. Signal transduction, proliferation, differentiation, and metabolism are all controlled by the balance of activity of protein kinases and protein phosphatases upon pivotal target proteins. Phosphorylation site prediction revealed 22 potential phosphorylation sites in PICP22, including 2 potential tyrosine phosphorylation sites. Tyrosine phosphorylation is involved in the replication of several herpesviruses (Geiss et al., 2001; Ren et al., 2001) and in shifting protein translocation from the cytoplasm to the nucleus during productive virus infection (Pomeranz and Blaho, 1999). Phosphorylation of VZV ORF63 is associated with its subcellular localization and transcriptional regulatory properties (Habran et al., 2005; Mueller et al., 2010), and phosphorylation of ICP22 is involved in HSV-1 virulence (Brandt and Kolb, 2003). Therefore, PICP22 phosphorylation may also play an important role during PRV infection, perhaps in modulating its subcellular localization or other uncharacterized functions, such as transcriptional regulation.

Nevertheless, hydrophobicity analysis indicated no strong hydrophobic region, although there were 6 hydrophobic regions principally situated in the intermediate region of the polypeptide chain. Sequence analysis showed no potential transmembrane domain, signal peptide, or N-linked glycosylation site (Asn-X-Ser/Thr), which may provide some clues regarding its expression. Secondary structure prediction (Figure 7) revealed that PICP22 pre- 
dominantly contained random coils and $\alpha$-helices. PICP22 also contained a coiled-coil motif, which is conserved in alphaherpesviruses (Pelletier et al., 1997; Mason and Arndt, 2004; Barbara et al., 2007) and mediates crucial protein-protein interactions, such as transcriptional control. Therefore, PICP22 might be a transcriptional factor that interacts with important host and virus proteins via the coiled-coil domain.

As more information becomes available on protein antigens, it should be possible to use this information to predict the locations of antigenic determinants prior to immunological testing. However, the elucidation of protein antigenic structures is presently a difficult, uncertain, and time-consuming task. Earlier methods were based on the assumption that the antigenic region is primary the hydrophilic region at the surface of the protein molecule (Hopp and Woods, 1981; Welling et al., 1985). However, this assumption is limited in its accuracy. To improve accuracy, the B-cell epitopes of PICP22 were predicted using DNAStar PROTEAN programs based on flexibility, antigenic index and surface probability based on the primary structure (Figure 7A). These findings of the antigenic and structural properties of PICP22 may yield methods for developing new antibodies and immunoassays for clinical diagnosis of PRV.

In the course of evolution, viruses are generally conserved and only a few genes undergo mutation (Antunes et al., 2010). Thus, viral evolution can be discussed at the molecular level. Our analysis revealed that the nucleotide sequence similarity of US1 of PRV Becker to Kaplan and Bartha was 92 and 89\% (Figures 2A and 3A); the aa sequence similarities were up to 92 and $88 \%$, respectively, consistent with a previous report (Szpara et al., 2011). Meanwhile, it may be that this evolutionary difference produces the variable virulence of distinct PRV strains. Multiple nucleotide and aa sequence alignments of PICP22 and the ICP22-like proteins showed that PICP22 has greater homology to members of Varicellovirus, especially EHV-1 (44.7 and 38.8\%, respectively). Therefore, PICP22 has relatively high homology to Varicellovirus, but not Simplexvirus, Mardivirus, or Iltovirus. Phylogenetic analysis (Figure 4) unequivocally demonstrated that PRV belongs to the genus Varicellovirus of subfamily Alphaherpesvirinae, consistent with a previous report (McGeoch et al., 2000). Moreover, PRV shares a closer evolutionary relationship with members of genus Varicellovirus than with other genera of Alphaherpesvirinae. Although the US1 gene products are conserved within the Alphaherpesvirinae subfamily, they are not conserved in other herpesvirus subfamilies, including Betaherpesvirinae and Gammaherpesvirinae, suggesting that the ICP22-like protein may execute a function that is discrete or complemented by other proteins from these subfamilies.

In conclusion, we presented the cloning and molecular characterization of the PRV US1 gene. Elucidating the molecular characterization and genetic evolution of PRV US1 will contribute to our understanding of this virus at the molecular level and will enrich the herpesvirus database. These studies will also provide insights for further research into the function and mechanism of USI during PRV infection.

\section{ACKNOWLEDGMENTS}

Research supported by grants from the Medical Scientific Research Foundation of Guangdong Province, China (\#B2012165); the National Natural Science Foundation of China (\#31200120); the Scientific Research Foundation for the Ph.D., Guangzhou Medical University (\#2011C20), and the Guangzhou City-Level Key Disciplines and Specialties of Immunology (\#B127007). We thank Dr. Lynn W. Enquist for the generous gift of pBecker2. 


\section{REFERENCES}

Advani SJ, Weichselbaum RR and Roizman B (2003). Herpes simplex virus 1 activates cdc2 to recruit topoisomerase II alpha for post-DNA synthesis expression of late genes. Proc. Natl. Acad. Sci. U. S. A. 100: 4825-4830.

Ambagala AP and Cohen JI (2007). Varicella-Zoster virus IE63, a major viral latency protein, is required to inhibit the alpha interferon-induced antiviral response. J. Virol. 81: 7844-7851.

Antunes RS, Gomes VN, Prioli SM, Prioli RA, et al. (2010). Molecular characterization and phylogenetic relationships among species of the genus Brycon (Characiformes: Characidae) from four hydrographic basins in Brazil. Genet. Mol. Res. 9: 674-684.

Barbara KE, Willis KA, Haley TM, Deminoff SJ, et al. (2007). Coiled coil structures and transcription: an analysis of the S. cerevisiae coilome. Mol. Genet. Genomics 278: 135-147.

Bastian TW, Livingston CM, Weller SK and Rice SA (2010). Herpes simplex virus type 1 immediate-early protein ICP22 is required for VICE domain formation during productive viral infection. J. Virol. 84: 2384-2394.

Bowman JJ, Orlando JS, Davido DJ, Kushnir AS, et al. (2009). Transient expression of herpes simplex virus type 1 ICP22 represses viral promoter activity and complements the replication of an ICP22 null virus. J. Virol. 83: 8733-8743.

Brandt CR and Kolb AW (2003). Tyrosine 116 of the herpes simplex virus type 1 IEalpha22 protein is an ocular virulence determinant and potential phosphorylation site. Invest. Ophthalmol. Vis. Sci. 44: 4601-4607.

Brukman A and Enquist LW (2006). Pseudorabies virus EP0 protein counteracts an interferon-induced antiviral state in a species-specific manner. J. Virol. 80: 10871-10873.

Coller KE, Lee JI, Ueda A and Smith GA (2007). The capsid and tegument of the alphaherpesviruses are linked by an interaction between the UL25 and VP1/2 proteins. J. Virol. 81: 11790-11797.

Feng ZP (2002). An overview on predicting the subcellular location of a protein. In Silico Biol. 2: 291-303.

Ferrari M, Gualandi GL, Corradi A, Monaci C, et al. (2000). The response of pigs inoculated with a thymidine kinasenegative (TK-) pseudorabies virus to challenge infection with virulent virus. Comp. Immunol. Microbiol. Infect. Dis. 23: $15-26$.

Geiss BJ, Tavis JE, Metzger LM, Leib DA, et al. (2001). Temporal regulation of herpes simplex virus type 2 VP22 expression and phosphorylation. J. Virol. 75: 10721-10729.

Habran L, Bontems S, Di VE, Sadzot-Delvaux C, et al. (2005). Varicella-zoster virus IE63 protein phosphorylation by roscovitine-sensitive cyclin-dependent kinases modulates its cellular localization and activity. J. Biol. Chem. 280: 29135-29143.

Hopp TP and Woods KR (1981). Prediction of protein antigenic determinants from amino acid sequences. Proc. Natl. Acad. Sci. U. S. A. 78: 3824-3828.

Jahedi S, Markovitz NS, Filatov F and Roizman B (1999). Colocalization of the herpes simplex virus 1 UL4 protein with infected cell protein 22 in small, dense nuclear structures formed prior to onset of DNA synthesis. J. Virol. 73: 51325138.

Jones JO and Arvin AM (2005). Viral and cellular gene transcription in fibroblasts infected with small plaque mutants of varicella-zoster virus. Antiviral Res. 68: 56-65.

Kalamvoki M and Roizman B (2011). The histone acetyltransferase CLOCK is an essential component of the herpes simplex virus 1 transcriptome that includes TFIID, ICP4, ICP27, and ICP22. J. Virol. 85: 9472-9477.

Kost RG, Kupinsky H and Straus SE (1995). Varicella-zoster virus gene 63: transcript mapping and regulatory activity. Virology 209: 218-224.

Kramer T, Greco TM, Enquist LW and Cristea IM (2011). Proteomic characterization of pseudorabies virus extracellular virions. J. Virol. 85: 6427-6441.

Krautwald M, Maresch C, Klupp BG, Fuchs W, et al. (2008). Deletion or green fluorescent protein tagging of the pUL35 capsid component of pseudorabies virus impairs virus replication in cell culture and neuroinvasion in mice. J. Gen. Virol. 89: 1346-1351.

Kyte J and Doolittle RF (1982). A simple method for displaying the hydropathic character of a protein. J. Mol. Biol. 157: 105-132.

Li M, Wang S, Cai M, Guo H, et al. (2011a). Characterization of molecular determinants for nucleocytoplasmic shuttling of PRV UL54. Virology 417: 385-393.

Li M, Wang S, Cai M and Zheng C (2011b). Identification of nuclear and nucleolar localization signals of pseudorabies virus (PRV) early protein UL54 reveals that its nuclear targeting is required for efficient production of PRV. J. Virol. 85: 10239-10251.

Lin HW, Hsu WL, Chang YY, Jan MS, et al. (2010). Role of the UL41 protein of pseudorabies virus in host shutoff, pathogenesis and induction of TNF-alpha expression. J. Vet. Med. Sci. 72: 1179-1187. 
Luxton GW, Lee JI, Haverlock-Moyns S, Schober JM, et al. (2006). The pseudorabies virus VP1/2 tegument protein is required for intracellular capsid transport. J. Virol. 80: 201-209.

Mason JM and Arndt KM (2004). Coiled coil domains: stability, specificity, and biological implications. Chembiochem 5: $170-176$.

McGeoch DJ, Dolan A and Ralph AC (2000). Toward a comprehensive phylogeny for mammalian and avian herpesviruses. J. Virol. 74: 10401-10406.

Mueller NH, Walters MS, Marcus RA, Graf LL, et al. (2010). Identification of phosphorylated residues on varicella-zoster virus immediate-early protein ORF63. J. Gen. Virol. 91: 1133-1137.

Nixdorf R, Klupp BG and Mettenleiter TC (2001a). Restoration of function of carboxy-terminally truncated pseudorabies virus glycoprotein B by point mutations in the ectodomain. J. Virol. 75: 11526-11533.

Nixdorf R, Klupp BG and Mettenleiter TC (2001b). Role of the cytoplasmic tails of pseudorabies virus glycoproteins B, $\mathrm{E}$ and $\mathrm{M}$ in intracellular localization and virion incorporation. J. Gen. Virol. 82: 215-226.

Orlando JS, Balliet JW, Kushnir AS, Astor TL, et al. (2006). ICP22 is required for wild-type composition and infectivity of herpes simplex virus type 1 virions. J. Virol. 80: 9381-9390.

Pelletier A, Do F, Brisebois JJ, Lagace L, et al. (1997). Self-association of herpes simplex virus type 1 ICP35 is via coiledcoil interactions and promotes stable interaction with the major capsid protein. J. Virol. 71: 5197-5208.

Pomeranz LE and Blaho JA (1999). Modified VP22 localizes to the cell nucleus during synchronized herpes simplex virus type 1 infection. J. Virol. 73: 6769-6781.

Ren X, Harms JS and Splitter GA (2001). Tyrosine phosphorylation of bovine herpesvirus 1 tegument protein VP22 correlates with the incorporation of VP22 into virions. J. Virol. 75: 9010-9017.

Smith GA and Enquist LW (2000). A self-recombining bacterial artificial chromosome and its application for analysis of herpesvirus pathogenesis. Proc. Natl. Acad. Sci. U. S. A. 97: 4873-4878.

Szpara ML, Tafuri YR, Parsons L, Shamim SR, et al. (2011). A wide extent of inter-strain diversity in virulent and vaccine strains of alphaherpesviruses. PLoS Pathog. 7: e1002282.

Tomioka Y, Miyazaki T, Taharaguchi S, Yoshino S, et al. (2008). Cerebellar pathology in transgenic mice expressing the pseudorabies virus immediate-early protein IE180. Eur. J. Neurosci. 27: 2115-2132.

Walters MS, Kyratsous CA, Wan S and Silverstein S (2008). Nuclear import of the varicella-zoster virus latency-associated protein ORF63 in primary neurons requires expression of the lytic protein ORF61 and occurs in a proteasomedependent manner. J. Virol. 82: 8673-8686.

Welling GW, Weijer WJ, van der Zee R and Welling-Wester S (1985). Prediction of sequential antigenic regions in proteins. FEBS Lett. 188: 215-218.

Xing J, Wang S, Lin F, Pan W, et al. (2011). Comprehensive characterization of interaction complexes of herpes simplex virus type 1 ICP22, UL3, UL4, and UL20.5. J. Virol. 85: 1881-1886.

Zhang G and Leader DP (1990). The structure of the pseudorabies virus genome at the end of the inverted repeat sequences proximal to the junction with the short unique region. J. Gen. Virol. 71: 2433-2441. 